\title{
Análisis de la Logística Hospitalaria aplicada en las Entidades de Salud de Nivel 3 y 4 en la ciudad de Barranquilla
}

\author{
Hospital Logistics analysis applied in the Level 3 and 4 Health Care \\ Institutions in the city of Barranquilla
}

\author{
Lindsay Figueroa Geraldino, Silebis Aguirre Lasprilla, María Wilches Arango, Daniel Romero \\ Rodríguez \\ Programa de Ingeniería Industrial, Universidad Autónoma del Caribe, Barranquilla, Colombia \\ Correo-e: lindsay.figueroa@uac.edu.co, silebis@hotmail.com,mwilches@uac.edu.co, \\ daniel.romero37@uac.edu.co
}

\begin{abstract}
Resumen- Este artículo presenta una metodología de tipo exploratoria descriptiva basada en el análisis de la logística hospitalaria desde sus diferentes características para las clínicas, IPS y hospitales de nivel 3 y 4 de la ciudad de Barranquilla. Este análisis profundiza sobre las áreas en la que se han aplicado herramientas de logística para el manejo de recursos. La principal debilidad encontrada en este sector es el manejo de inventarios debido a múltiples dificultades sobre abastecimiento y optimización de los niveles adecuados para el funcionamiento eficiente. Es por ello, que a través de esta investigación se establece la situación actual de las entidades de la ciudad de Barranquilla con el fin de proponer recomendaciones que le permitan al sector salud mejorar las condiciones presentes.
\end{abstract}

En esta investigación se aplicó una encuesta a entidades de salud a partir de la cual se obtuvo como resultado que al momento de implementar una herramienta logística existen limitantes financieras, físicas y tecnológicas. Además, se identificó que el manejo y control de inventarios es la herramienta logística que más se ha implementado, aun cuando las entidades consideran que aún se debe ser reforzada.

Palabras clave - Entidades de Salud, Manejo de inventarios, logística hospitalaria, Sistemas de inventarios.

Abstract- This article shows a methodology of descriptive exploratory type based on the analysis of hospital logistics from their different characteristics for clinics, Level 3 and 4 hospitals and IPS of the city of Barranquilla. This analysis delves into the areas in which they have applied logistics tools for managing their resources. The main weakness found in this sector is the inventory management due to its many difficulties on supply and optimization of appropriate levels for the efficient operation. That's why, through this research is established the current situation of entities in the city of Barranquilla to propose recommendations to enable the health sector to improve the present conditions.

In this research a survey was applied to health care institutions from which it was obtained as a result that when implementing a logistical tool there are financial, physical and technological constraints. In addition, it was identified that the management and control of inventories is the logistics tool most widely implemented, even when the entities consider that it still needs to be strengthened.

Key Word - Health Care Institutions, Hospital Logistics, Inventory Management, Inventory Systems.

\section{INTRODUCCIÓN}

La logística hospitalaria permite utilizar técnicas computacionales con el fin de facilitar la cadena de suministros de los procesos logísticos de un Centro de salud, a partir de las cuales se pueden recorrer las actividades desde el fabricante hasta el paciente. Es un tema que ha venido en evolución y en la actualidad existen muchas clínicas y hospitales que implementan las herramientas logísticas para encontrar oportunidades de mejora y prestar un mejor servicio a los pacientes.

En los centros de servicios médicos, la logística hospitalaria se ha centrado en gran parte en la gestión de inventarios, buscando desarrollar nuevas técnicas a través de las que se mejora en gran 
parte la eficiencia de la organización y aumentar la satisfacción del cliente vista desde el bienestar del mismo.

Es importante indicar que la información en Colombia sobre la aplicación de la Logística en clínicas y hospitales es escasa, por lo que no se tiene claridad sobre los costos que acarrean las actividades logísticas. La mayoría de los gastos de las instituciones hospitalarias, se realizan en actividades diferentes a la atención de pacientes y prevención. Ordoñez, L \& Bustamante, A (2011).

Los objetivos de esta investigación son desarrollar un diagnóstico acerca de la logística hospitalaria para la construcción de un estado del arte con el fin de mostrar la aplicación de herramientas logísticas en las IPS, Clínicas y Hospitales de nivel 3 y 4 de la Ciudad de Barranquilla y establecer un plan de acción para proponer mejoras en el ámbito de la logística hospitalaria en los centros de salud de la ciudad.

\section{CONTENIDO}

\section{A. ESTADO DEL ARTE.}

La logística hospitalaria es una herramienta que permite el manejo adecuado de recursos, integra todas las actividades desde la obtención de materia prima, transformación, almacenaje, distribución final, hospitalización y salida del paciente, es por ello que se considera un aspecto relevante porque determina el funcionamiento de procesos internos a partir de los cuales se pueden proponer alternativas de mejora.

La óptima gestión de las labores logísticas hospitalarias, es un factor determinante para reducir costos y obtener una alta calidad en la atención sanitaria. [1]

El mal uso de los recursos evidencia un desequilibrio económico, que no ocurriera si se implementaran acciones correctas. Esto es una consecuencia directa del desconocimiento del funcionamiento hospitalario, lo que conduce a que gran parte de las actividades logísticas sean compartidas [2]

Beaulieu y Landry [3] enuncian que otra de las consecuencias del mal uso es que los responsables de las actividades logísticas están alejados de los principales centros de decisión del establecimiento y que buscan sobre todo respetar los procedimientos más que mejorarlos

La logística hospitalaria tiene dos puntos claves, la gestión de inventarios, sobre la cual se enfocará este artículo y la programación de recursos, que busca conocer la disposición de los productos que se encuentran almacenados en el hospital, abarcando tanto la programación de medicamentos como la planificación de médicos, habitaciones y las salas de cirugía.

La gestión de inventarios busca controlar el nivel y costo del mismo, principal problema que aqueja las entidades de salud, tiene en cuenta principalmente la capacidad que tiene el hospital para el almacenaje del medicamento y otros elementos como los pacientes, sus necesidades, la capacidad de inventario y los diferentes puntos donde se encuentren los insumos, etc.

Amaya, et al. en su artículo potenciando la contribución de la logística hospitalaria: tres casos, tres trayectorias, realizan un estudio a hospitales en Francia, Canadá y Colombia que han aplicado logística hospitalaria reflejando para el caso de Clínica Saint Martín (Francia) ciertas dificultades en el reabastecimiento de los productos en cada unidad; para el Hospital Sacré (Canadá) el problema estaba más enfocado en la falta de automatización, realización de actividades logísticas de manera independiente y alta ocupación del personal médico; y para el Hospital el Tunal (Colombia) se encontraron debilidades en las áreas de lavandería, farmacia y urgencias con mayor relevancia en la primera por las demoras de sus actividades [2].

Las soluciones propuestas para cada uno fueron implementar un sistema de doble cajón, aplicar automatización y por último se propuso un modelo de programación lineal entera que determinaría la frecuencia de la recolección de ropa sucia y a partir de ahí generar las rutas para mejorar la eficiencia del recorrido.

De igual manera se concluye que el contexto no influye directamente sobre la contribución logística, pero estimula a los directivos a emprender proyectos de mejora a los hospitales. Además, aseguran que es importante la integración de las tecnologías de la información debido al auge del comercio electrónico.

Por otro lado, uno de los factores más difícil de cuidar integralmente es el problema de los sistemas de inventarios, el cual se presenta en muchos hospitales evidenciando multiplicidad de causas relacionadas con la falta de control gerencial hasta falta de automatización, que permita una interrelación entre farmacias y bodegas.

Hernández, Velasco \& Amaya C. [4] realizan un proyecto de coordinación de la cadena de abastecimiento incorporando un sistema de inventarios multinivel con $\mathrm{M}$ - proveedores, 1Bodega y $\mathrm{N}$-minoristas en un hospital público. Estos sistemas multinivel observan la cadena de suministros como un todo, teniendo en cuenta la influencia de un nivel sobre otro respecto al inventario 
Ballou R. [5] asegura que, si se mantienen cantidades sustanciales de inventario en los almacenes de campo, entonces pueden necesitarse menos en el siguiente nivel inferior del canal

Este hospital público cuenta con 800 insumos farmacéuticos, 46 proveedores, un centro de almacenamiento (bodega) y 6 farmacias (bodegas descentralizadas). La existencia de faltantes, acarrea costos adicionales y maximiza los costos de ordenar y mantener el inventario, es por ello que a través de un modelo heurístico se propone optimizar la cantidad según la demanda.

La formulación matemática se realiza mediante métodos de búsqueda local y algoritmos genéticos que son modelos heurísticos para facilitar la resolución del problema. Los primeros son algoritmos iterativos que parten de una solución inicial para moverse dentro de un espacio de búsqueda definido. Los algoritmos genéticos, que están basados en operadores que simulan procesos naturales de evolución, manejan los operadores para producir nuevas generaciones, que permitan encontrar soluciones más acertadas para la función objetivo.

Gracias a la naturaleza determinística de la demanda, el modelo arrojó cantidades de lotes exactos. El modelo permite reducir hasta el $32 \%$ de los costos totales incurridos por el área farmacéutica. Los autores recomiendan al área de farmacia considerar tiempos de reabastecimiento para cada bodega dependiendo de las características de cada proveedor y así analizar los tiempos de reposición de inventario para las áreas de mayor dificultad, en este caso farmacias de urgencia y cirugía.

Por otro lado, Bustamante \& López [6], analizan el efecto cola en una cadena de distribución a un hospital tipo 1 ubicado en Chile. Este se realizó en las redes de prestación de salud que determinó el Servicio Nacional de Salud, creadas con el fin de mejorar la calidad del servicio hospitalario involucrando al paciente en la Medicina Preventiva. Dentro del estudio se sustenta que el hospital no cuenta con un registro de los insumos y materiales utilizados por cada uno de los pacientes de los pedidos por mes. El hospital tampoco cuenta con un registro de pedidos extraordinarios y de urgencia para el manejo de contingencias.

A través de un gráfico de control, se evidencia que el número de solicitudes efectuadas por farmacia es mucho mayor que la solicitada por otros servicios. El estudio buscó estimar el tamaño del inventario a través de un prototipo en Excel que calculara la demanda real por insumos comparada con el inventario. El prototipo integra todos los servicios para que el sistema de abastecimiento ocurra en el menor tiempo posible, haciendo que la información se entregue en línea y no se tomen como departamentos independientes (sistema bullwhip), lo cual es causal del retraso.

Con este sistema o cualquier otro que centralice la información de los insumos y el uso de los mismos, se puede reducir el inventario hasta en un $40 \%$. La implementación del sistema necesita de personas que estén abiertas a los cambios y transformaciones.

La importancia de tener claridad en todo el proceso de compra, facturación, políticas de calidad, objetivos, metas a mediano y corto plazo es esencial para el funcionamiento de los centros de salud. Con base a los servicios ofrecidos por la entidad, se debe tener todo un sistema que funcione adecuadamente para que no se vea afectada la vida del paciente.

Todo esto se puede dar siempre y cuando se le apliquen mejoras a la cadena de suministros y por supuesto acreditando a la logística como la herramienta adecuada para el desarrollo de las operaciones presentadas dentro y fuera del hospital, y sobre todo satisfaciendo la necesidad del paciente ya que siempre se le va garantizar la disponibilidad de lo que dicho paciente necesite con respecto a lo que su enfermedad o emergencia amerite.

Ya con el pasar del tiempo no solo se han enfocado en las anteriores áreas expuestas, también existen servicios relacionados con las necesidades básicas prestadas (alimentación, lavandería, mantenimiento, aseo, etc.), que necesitan mejoras significativas ya que algunas de ellas presentan dificultades que requieren del uso de las herramientas de la logística hospitalaria sin abandonar la labor original de la entidad prestadora del servicio. Por ejemplo, el estado adecuado de las instalaciones donde se va a prestar el servicio, el aseo adecuado para evitar contaminaciones, las habitaciones en excelentes condiciones para albergar el paciente, la comida necesaria para cada paciente y justa en el momento requerido. Todo lo anterior afecta los servicios brindados al paciente, consultas y cirugías [7]

La innovación constante en la logística hospitalaria y las posibles aplicaciones de este en un hospital debe siempre ser importante para una entidad, siempre enfocándose en el cómo puede beneficiar la cadena de suministros y la relación con los proveedores, estableciendo que estos se dé la mejor manera posible y se puedan apreciar los frutos de un buen uso de la innovación en la logística hospitalaria para una entidad de salud. [8]

Los elementos teóricos a destacar de esta revisión bibliográfica es que en Colombia si hay evidencia de implementación de 
herramientas logísticas, sin embargo, no existe evidencia teórica suficiente que sustente que las Entidades de Salud de Ciudad de Barranquilla implementen herramientas de logística hospitalaria. La gestión de inventarios es uno de los pilares de la logística hospitalaria y en ella es importante la relación que pueda existir con el proveedor y el comprador, porque la farmacia debe considerar tiempos de reabastecimiento para cada bodega y así poder analizar los tiempos de reposición de inventario.

Las entidades de Salud deben contar con un sistema que centralice la información de la entrada de insumos. Esto podría generar que se reduzca el inventario y con ello los costos asociados. Generalmente un buen manejo gerencial y la automatización son las claves para una verdadera gestión de inventarios.

\section{METODOLOGIA}

Este proyecto se realizó en tres fases, desarrolladas de la siguiente forma:

- FASE 1: revisión de la literatura existente sobre Logística Hospitalaria, con el fin de extraer elementos teóricos que sustentarán la base de este proyecto.

- FASE 2: Se realizó un análisis a las IPS, Clínicas y Hospitales de nivel 3 y 4 de la Ciudad de Barranquilla sobre el manejo de logística hospitalaria especialmente para examinar acerca de las herramientas empleadas en cada uno de estos Centros de salud.

Para poder llevar a cabo el estado de la situación actual de cada Entidad prestadora de salud respecto a esta temática, se utilizó la encuesta como principal fuente de recolección.

Se identificaron 37 entidades prestadoras de salud que se encuentran en este rango. Inicialmente se encontró el tamaño de la muestra, al cual se le aplicó un nivel de confianza del 5\% con un error del $10 \%$ y se encontró, como se muestra a continuación, que el tamaño de la muestra debían ser 25 entidades a encuestar.

$$
\begin{gathered}
n=\frac{Z_{\alpha}{ }^{2} \times N \times p \times q}{i^{2}(N-1)+Z_{\alpha}{ }^{2} \times p \times q} \\
n=\frac{(1,96)^{2} \times 33 \times 0,5 \times 0,5}{0,1^{2}(33-1)+(1,96)^{2} \times 0,5 \times 0,5} \\
n=24,75 \approx 25
\end{gathered}
$$

De estas 25 entidades prestadoras de salud, 20 respondieron la encuesta y 5 no dieron información, a pesar que se indicó el manejo de la confidencialidad.

Las personas que respondieron esta encuesta en su mayoría era personal administrativo, de calidad o de logística.

- FASE 3: Con base a las respuestas encontradas en el diagnóstico de la situación actual de las entidades y con los elementos teóricos extraídos de la revisión bibliográfica, se elaboró una serie de conclusiones con el objeto de crear un plan de acción que le permita a las clínicas, hospitales e IPS mejorar puntos importantes en el manejo de su logística hospitalaria.

\section{DIAGNOSTICO}

Antes de comenzar con los resultados que arrojó la encuesta, es importante contextualizar y delimitar el estudio, con el fin de conocer cómo se encuentra la ciudad con referencia al tema de la salud. Barranquilla es la capital del Departamento del Atlántico en Colombia. Este se encuentra limitado al oriente con el rio Magdalena y al norte y noroeste por el Mar Caribe, por estas razones constitucionalmente se le conoce como Distrito especial, industrial y portuario. Al ser una ciudad costera su principal actividad económica es el comercio.

Barranquilla cuenta con $154 \mathrm{~km} 2$ y además del comercio se realizan distintas actividades y se ha evidenciado cómo el clúster de la salud ha crecido a pesar de ser una ciudad tan pequeña y de los problemas en temas de corrupción y desvíos de fondos que se han dado en este sector, ha surgido como una estrategia empresarial para poder competir en el mercado internacional. Según la Organización Mundial de la Salud en el 2007 Colombia es el país de Latinoamérica que más gasta en salud con un porcentaje del 6,1\% en el año 2007, después de Argentina y Brasil.

En el siguiente grafico se evidencia el $85,5 \%$ de los recursos públicos son destinados para el sector Salud a diferencia de los demás países latinoamericanos. 


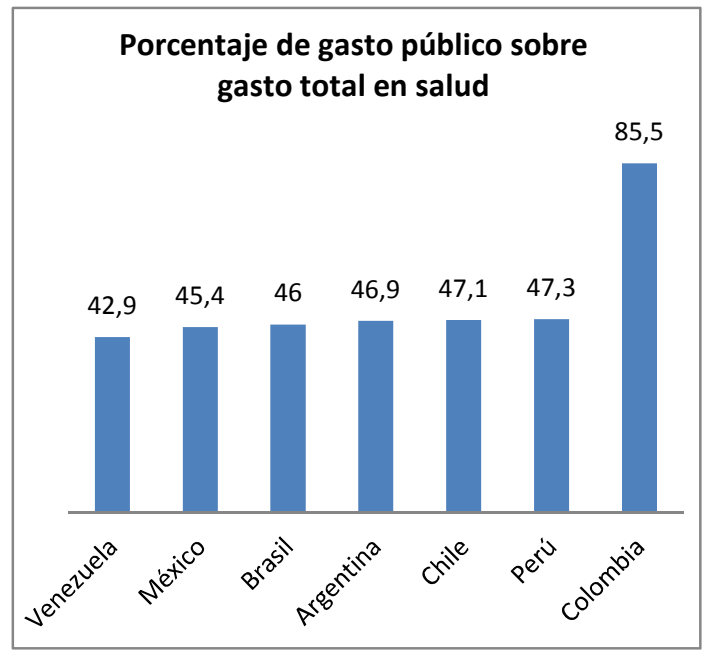

Figura 1. Porcentaje de gasto público sobre gasto total en salud. Fuente: OMS, 2014

Específicamente refiriéndose a la ciudad de Barranquilla, en cuanto al clúster de Salud, la mayor actividad es la prestación de servicios de salud que corresponde a clínicas especializadas, clínicas generales, consultorios, hospitales, centros de diagnóstico, laboratorios clínicos, etc. Como segunda actividad se encuentra la distribución de productos farmacéuticos y equipamiento médico y por último la actividad de los proveedores de insumos.

Barranquilla es una de las ciudades con la mayor densidad de IPS privadas, ya que cuenta con 22 IPS por cada 100.000 habitantes comparada con Cali, Medellín y Bogotá tal como lo muestra la Tabla 1.

\begin{tabular}{|l|l|l|l|}
\hline CIUDAD & ESE & $\begin{array}{l}\text { IPS } \\
\text { Privadas }\end{array}$ & $\begin{array}{l}\text { Tasa } \\
100.000 \\
\text { habitantes }\end{array}$ \\
\hline Barranquilla & 3 & 169 & 22 \\
\hline Bogotá & 24 & 1365 & 19 \\
\hline Cali & 10 & 411 & 19 \\
\hline Medellin & 4 & 394 & 17 \\
\hline
\end{tabular}

Tabla 1. Cantidad de IPS privadas por habitante. Fuente: OMS, 2014

Es importante acotar que a pesar de esa ventaja igual no se cuentan con los suficientes profesionales independientes, tal como lo muestra la Tabla 2.

\begin{tabular}{|l|l|l|}
\hline CIUDAD & $\begin{array}{l}\text { Profesionales } \\
\text { Independientes }\end{array}$ & $\begin{array}{l}\text { Tasa } \\
100.000 \\
\text { habitantes }\end{array}$ \\
\hline Barranquilla & 404 & 35 \\
\hline Bogotá & 7384 & 105 \\
\hline
\end{tabular}

\begin{tabular}{|l|l|l|}
\hline Cali & 1675 & 77 \\
\hline Medellin & 3170 & 140 \\
\hline
\end{tabular}

Tabla 2. Cantidad de profesionales independientes por habitante. Fuente: OMS, 2014

Si se observa dentro de la capacidad instalada del Sector Salud en Barranquilla, se puede resaltar la oferta de Camas que posee la ciudad teniendo en cuenta la tasa de habitantes, lo cual nos da una ventaja competitiva con respecto a esas ciudades principales en cuestión.

\begin{tabular}{|l|r|l|}
\hline Ciudad & No. Camas & $\begin{array}{l}\text { Camas x 10000 } \\
\text { Hab }\end{array}$ \\
\hline Barranquilla & 2805 & 24,1 \\
\hline Medellin & 4451 & 19,8 \\
\hline Cali & 3501 & 16,5 \\
\hline Bogota & 9200 & 13,4 \\
\hline
\end{tabular}

Tabla 3. Densidad del número de camas en las ciudades principales de Colombia.

Fuente: OMS, 2014

Según la Organización Mundial de la Salud en este indicador de camas, entre el 2006 y 2012 en países con niveles de ingreso medio alto, incluido Colombia fue de 32 camas hospitalarias por cada 10,000 habitantes, específicamente Colombia presento 15 en total [9].

En el año 2010 se realizó un análisis de la suficiencia de la Red Prestadora de Servicios de Salud en el Departamento del Atlántico y Distrito de Barranquilla el cual mostro como resultado la capacidad instalada del Departamento como tal distribuido en Nodos (Barranquilla, Soledad y Sabanalarga) incluyendo en cada uno de ellos los municipios, que puntualmente se contaba con 1.484 camas de hospitalización para adultos, $72 \%$ hacen parte de las entidades prestadoras privadas y $28 \%$ de la Publica. Esto genera una instancia de 541.660 estancias disponibles para satisfacer un total de 1.446.319 adultos que demandarían 113.763 ingresos al año, es decir 9.480 en promedio mensual. La tasa de ingreso mensual es de78,66 por cada 1000 adultos.

La estancia promedio es de 3,53 días, por lo que se estima que la población adulta demandara al año 401.049 estancias al año, dando como resultado que también haya una desproporción por nodos en relación a la población en comparación con la oferta de camas, ejemplo Barranquilla con una población de 60,58\% adulta y una oferta de camas de $85,24 \%$ a diferencia de Soledad 
con población adulta de $31 \%$, pero con capacidad instalada de $8,22 \%[10]$.

En la Tabla 4 para el 2013 que las camas de adulto eran 1758 para un total de 4058 camas como Registro de Capacidad Instalada del Segundo Cuatrimestre del Distrito, en el 2012 era una relación de 1.548 camas para un total 3,662 camas en general, dándose un incremento porcentual entre los dos años del $13,65 \%$ cama adulta y de $10,81 \%$ en total de camas.

El clúster de Salud es una estrategia para el desarrollo de la economía del Distrito pero desde el punto de vista innovativo y competitivo posee falencias coyunturales en sus procesos, productos y servicios, dentro de su capacidad instalada se evidencian debilidades por la insuficiencia de camas para atender la demanda de igual manera de profesionales, además de una falta de cultura organizacional basada en la omisión de planificación estratégica basado en fines y en el seguimiento oportuno de los procesos, afectando a las entidades de salud incluso en el ejercicio de sus tareas diarias.

\begin{tabular}{|l|r|r|}
\hline \multicolumn{3}{|c|}{ REGISTRO CAPACIDAD INSTALADA DISTRITO 2013} \\
\hline CAMAS INSCRITAS & I CUATRI & II CUATRI \\
\hline ADULTOS & 1722 & 1758 \\
\hline CUIDADO AGUDO MENTAL & 64 & 57 \\
\hline U.C.I ADULTO & 285 & 299 \\
\hline U.C.I PEDIATRICA & 54 & 54 \\
\hline U.C.I NEONATAL & 131 & 139 \\
\hline CUIDADO INTERMEDIO ADULTO & 139 & 139 \\
\hline CUIDADO INTERMEDIO MENTAL & 57 & 71 \\
\hline CUIDADO INTERMEDIO & & \\
PEDIATRICO & 25 & 25 \\
\hline CUIDADO INTERMEDIO & & \\
NEONATAL & 166 & 173 \\
\hline FARMACODEPENDENCIA & 56 & 56 \\
\hline OBSTETRICIAS & 364 & 363 \\
\hline PEDIATRICAS & 417 & 406 \\
\hline PSIQUIATRICAS & 177 & 155 \\
\hline UNIDAD DE QUEMADOS & & \\
ADULTOS & 22 & 10 \\
\hline UNIDAD DE QUEMADOS & & \\
PEDIATRICA & 14 & 8 \\
\hline SALAS DE PARTO & 38 & 37 \\
\hline QUIROFANOS & 213 & 220 \\
\hline AMBULANCIAS BASICAS & 51 & 55 \\
\hline AMBULANCIAS MEDICALIZADAS & 30 & 33 \\
\hline TOTAL & $\mathbf{4 0 2 5}$ & $\mathbf{4 0 5 8}$ \\
\hline
\end{tabular}

Tabla 4. Registro de Capacidad Instalada Distrito. Fuente: Secretaria Distrital de Salud, Barranquilla, 2013[10]

Teniendo en cuenta la información estructural en la Ciudad de Barranquilla que da una visión holista del desarrollo en el Sector Salud y las falencias puntuales en temas de logística hospitalaria, se aplica la encuesta en 20 Entidades de Salud de nivel 3 y 4 de la Ciudad de Barranquilla, debido a que 5 por temas de confidencialidad no aportaron información.

En el desarrollo de la encuesta se hizo énfasis inicialmente en el conocimiento sobre el tema y evidentemente la respuesta fue positiva en un $80 \%$; más sin embargo su conocimiento es basado en las generalidades que incluye la Logística Hospitalaria pero en realidad.

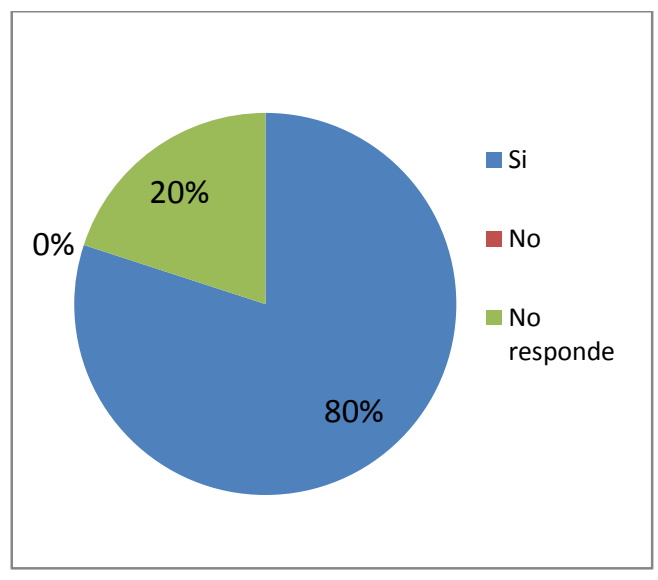

Figura 2. Conocimiento de las entidades sobre el término de logística hospitalaria.

Fuente: Elaboración propia.

Del resultado generado por la gráfica 2, cabe resaltar que algunas de estas entidades cuestionaban sobre el significado de logística hospitalaria antes de dar sus respuestas, justificando que no conocían el término como tal, pero si tenían conocimiento sobre su significado y las actividades que abarca. Por ello, la pregunta siguiente que hacía referencia a si se conocía algún proyecto asociado a la logística hospitalaria, el mismo $80 \%$ de los encuestados en cada entidad respondió positivamente.

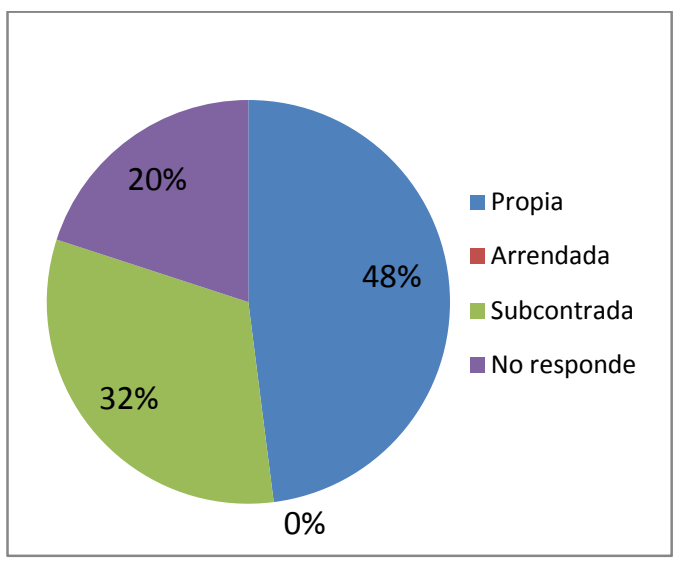

Figura 3. Tipo de farmacia de las entidades. Fuente: Elaboración propia. 
Por otro lado, la gráfica 3 muestra que la mayoría de las farmacias son propias con un porcentaje del $48 \%$, lo que genera una incidencia directa sobre el manejo y control de recursos. Muchas de las entidades que respondieron que tenían farmacia subcontratada comentaban que esto era una ventaja porque representa una mayor oportunidad de tener los medicamentos a tiempo y tener un mayor control sobre otras áreas del Centro de salud.

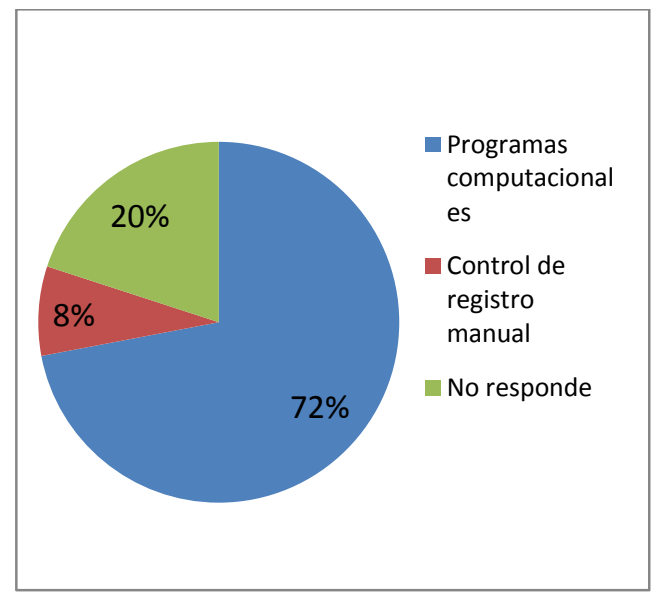

Figura 4. Forma de planeación de recursos requeridos efectuada por las entidades.

Fuente: Elaboración propia.

El gráfico 4 interrelaciona la pregunta sobre los métodos de política de inventarios y la forma como es manejado y controlado ese inventario. Aunque muchas de las entidades respondían que si existían políticas de inventario muchos no lo manejaban adecuadamente, incluso no todo el personal tenía el conocimiento para manejar el software implementado por la organización cuando esta contaba con uno en específico, otras pocas entidades que no contaban con el software respondían que no tenían procesos eficientes de manejo de inventarios, porque resulta más complicado la administración del mismo a través de los registros manuales debido a que les impide tener una actualización en tiempo real. En este gráfico se muestra que el $72 \%$ cuenta con un registro computarizado que les permite llevar un método exacto para el control de medicamentos e insumos.

Con respecto a los problemas de retrasos, muchas de las entidades a las cuales se les realizó la encuesta respondían con el término "Dentro de los términos normales". Esto significa que existe una probabilidad de no tener un medicamento cuando el paciente lo solicita, sobre todo si tenía una alta demanda o era de tipo especial básicamente por el trámite de la autorización.
La segunda parte muestra las respuestas a las preguntas que permiten indagar un poco más a fondo sobre los proyectos de logística hospitalaria que han efectuado y las que han dejado de realizar en cada una de las entidades. Estos son los puntos más relevantes de la encuesta porque a partir de ellos se podrán continuar proyectos futuros sobre el tema de logística hospitalaria para la ciudad de Barranquilla.

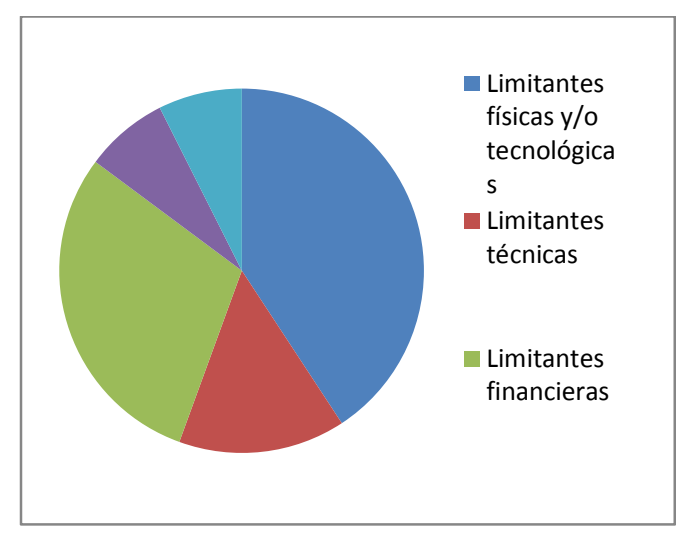

Figura 5. Limitantes obtenidas al aplicar un proyecto de logística hospitalaria.

Fuente: Elaboración propia

El gráfico 5 muestra que los mayores obstáculos en la implementación de algún proyecto asociado a la logística hospitalaria son las limitantes físicas y tecnológicas con un $41 \%$. Las razones por las cuales se escogió este inconveniente fueron porque en ocasiones las entidades crecían y no tenían el espacio físico para realizar los cambios y la parte tecnológica por la dificultad para mantenerse a la vanguardia con los mejores equipos y métodos. Esto es directamente proporcional con las limitantes financieras, ya que una expansión y los cambios en los equipos requieren tiempo, recursos $\mathrm{y}$ capacitaciones del personal que se convierten en dinero para las entidades prestadoras de salud.

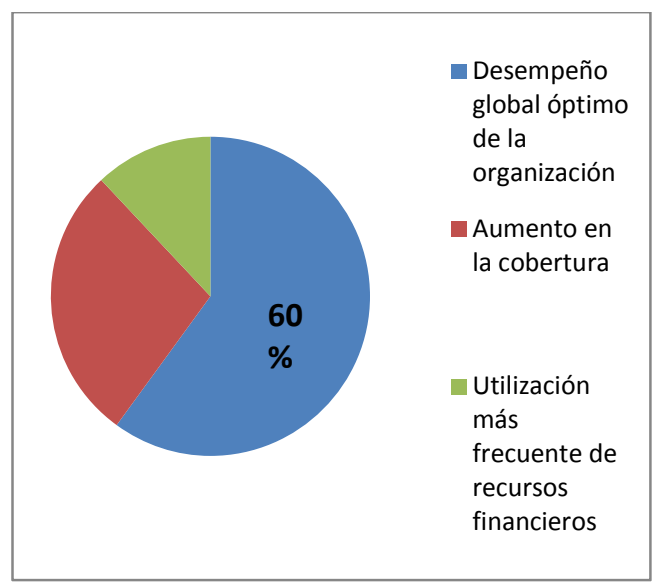

Figura 6. Resultados obtenidos al aplicar el proyecto de logística hospitalaria.

Fuente: Elaboración propia. 
El gráfico 6 representa lo que significa para las entidades implementar un proyecto de logística hospitalaria. El 60\% de las Entidades consideran que el implementar estos proyectos beneficia al desempeño global óptimo de la organización. El desempeño global óptimo lo que busca es la integralidad del sistema e interrelación entre las diferentes áreas del Centro de salud.

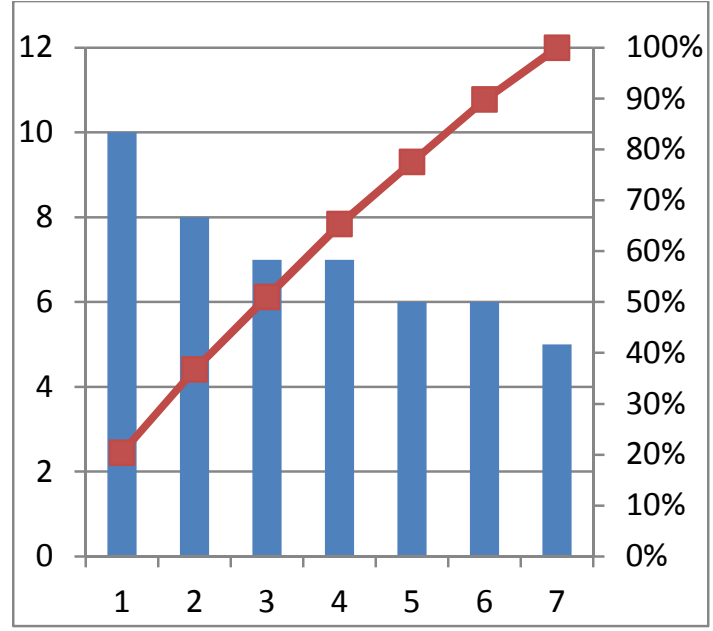

Figura 7. Áreas en que se ha aplicado herramientas de logística hospitalaria.

Fuente: Elaboración propia.

Donde,

1. Manejo y control de inventarios

2. Organización de bodegas

3. Diseño y distribución de áreas

4. Determinar capacidad de recursos

5. Programación de citas /cirugías

6. Análisis de la cadena de suministro

7. Planeación y programación del recurso humano

En el gráfico 7 se evidencia que el 20\% de las causas están concentradas en el manejo y control de inventarios, al igual que en el gráfico 5. Esto conlleva a que se realicen mayores esfuerzos en este primer ítem.

Por otro lado, según el nivel de frecuencias, la gráfica 4 aunque muestra que si se ha trabajado en la aplicación de herramientas de logística hospitalaria, la planeación y programación del recurso humano es lo que menos han considerado las entidades. Sin embargo, en la gráfica 5 toma fuerza la necesidad de comenzar a implementar mejoras en planeación en el recurso humano. Paralelo a ello se encuentra la programación de citas y cirugías, que aunque se ha aplicado el uso de esta herramienta, las entidades consideran que deben seguir haciéndose esfuerzos por hacerlas más eficientes.

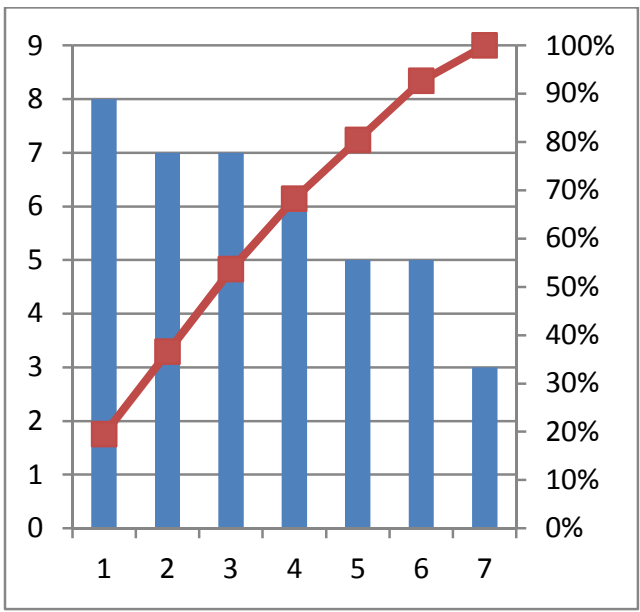

Figura 8. Áreas en las que se necesitan aplicar herramientas de logística hospitalaria.

Fuente: Elaboración propia.

Donde,

1. Manejo y control de inventarios

2. Programación de citas/cirugías

3. Organización de bodegas

4. Planeación y programación del recurso humano

5. Diseño y distribución de áreas

6. Análisis de la cadena de suministro

7. Determinar capacidad de recursos

En cuanto al diseño y distribución de áreas, muchas de las entidades de la ciudad de Barranquilla le han apostado a este aspecto con el fin de mejorar la comodidad, aumentando especialmente la satisfacción del cliente. Es por esta razón que los resultados al respecto en la gráfica 8 van enfocados para las entidades que no han implementado una mejora en la infraestructura, que aunque han querido realizarlo, no han podido ejecutarlo por limitantes financieras (Gráfico 5).

Determinar la capacidad de recursos es el aspecto que las entidades consideran que menos deben implementar y la razón principal que argumentan es que siempre han trabajado bajo el mismo esquema, con las mismas herramientas y equipos. Los Centros de salud han tenido grandes cambios a raíz de que implementaron herramientas logísticas.

Con respecto al análisis de la cadena de suministros que en las Entidades de Salud y Hospitales están relacionadas con los medicamentos e insumos, se observa que estas empresas dan por sentado esta herramienta porque existe una cultura organizacional que demanda el trabajo bajo un estándar, aunque no tienen muy claro la aplicabilidad y administración de este.

El manejo y control de inventarios y la organización de bodegas son los aspectos en los que más se han implementado 
herramientas logísticas, y que resulta importante para las Organizaciones implementarlas. Cuando nos referimos a la organización de bodegas es vital tener en cuenta la modalidad de propiedad que maneja la farmacia, debido a que, si es arrendada, la entidad suele no preocuparse por la manera como se maneja los recursos dentro de la bodega, siempre y cuando los insumos estén cuando se soliciten. Para el manejo y control de inventarios, las entidades argumentan que siempre será una dificultad poder controlar el inventario porque la demanda es inestable.

La logística hospitalaria está centrada en incrementar los niveles de servicio y crear satisfacción en los pacientes de tal manera que se pueda mejorar los procesos internos de los Centros de Salud en temas de organización, para esto es necesario contar una capacidad instalada propicia para mejorar los tiempos de atención oportunos. Básicamente en Colombia la logística hospitalaria es un tema aun inexplorado y se basan básicamente sus gastos en atender otros aspectos diferentes al fin último de esta que es la atención a pacientes de manera eficiente. Es vital que bajo este concepto se fortalezcan aspectos relacionados con la gestión de la cadena de abastecimiento global en las Clínicas y Hospitales de Nivel 3 y 4 en todo lo relacionado con temas de planeación, abastecimiento, entrega y gestión de retornos, ya sea en productos y/o servicios, para satisfacer la necesidad de los pacientes.

\section{B. PROPUESTAS DE MEJORA}

De acuerdo con la evaluación obtenida en el diagnostico se hace necesario definir una serie de estrategias, con la finalidad de establecer un apoyo en la gestión de insumos, la tecnología, la infraestructura y los equipos que requieren las Entidades de Salud estudiadas, de tal manera que respondan a las necesidades del Sector. El objetivo principal de la propuesta es que estas Entidades puedan implementar una herramienta logística en el área medico asistencial de diagnóstico que presente mayores dificultades a través de estrategias gerenciales y operacionales con el fin de disminuir costos innecesarios.

Algunas de las estrategias que se recomiendan son:

Estrategias

- Desarrollar un análisis de la demanda de insumos y medicamentos para establecer un método de pronósticos que se ajuste al comportamiento de la demanda de los suministros médicos utilizados por cada área
- Establecer un análisis ABC con el fin de identificar la rotación e impacto de los insumos y medicamentos que son almacenados, identificando de esta manera qué suministros son de alta y baja rotación y de bajo y alto impacto, la magnitud de las áreas ocupadas por estos.

- Definir Indicadores en las unidades médico asistenciales como manera de llevar un control de la logística interna y desempeño entre áreas que son clientes internos

- Desarrollar investigaciones de casos exitosos de otras entidades prestadoras de salud con el fin identificar herramientas logísticas que permita enriquecer la gestión hospitalaria en la Clínica, IPS u hospital

- Se deben realizar revisiones periódicas durante y después de la implementación y además buscar siempre innovar a través de la mejora continua "Kaizen", con ello se crean soluciones constantes y se mejora el desempeño.

- Implementar un sistema Kanban el cual consiste en la aplicación de una etiqueta para determinar el sistema reabastecimiento de insumos y medicamentos de acuerdo a la demanda y el nivel de inventario de los mismos. Con ello se garantiza la disponibilidad del insumo y se reduce el inventario.

- Desarrollar la técnica Jidoka de inspección automatizada realizada a través de pistolas lectoras de códigos de barras, lo que posibilita que los pedidos puedan ser controlados en tiempo real, evitando así riesgos manuales.

- Aplicar un sistema de inventario multinivel para mejorar coordinación de inventarios, que tiene en cuenta la influencia de un nivel sobre otro en la cadena de inventario y aliviar el efecto látigo para mantener así la misma disponibilidad de producto.

- Integrar las compras y centralizar la información a través de un prototipo de Excel para minimizar la cadena de distribución dependiente de un nivel de servicio, de tal manera que se reduzca el inventario hasta en un $40 \%$.

- Mantener una comunicación con los miembros de las entidades prestadoras de salud sobre el área en el cual desempeñan sus funciones y lograr que puedan comprometerse a efectuar los cambios que sean necesarios. 


\section{CONCLUSIONES}

En la ciudad de Barranquilla muchas Clínicas y Hospitales de nivel 3 y 4 aunque han implementado herramientas logísticas, aún están en la búsqueda de herramientas más eficaces en áreas como la organización de bodegas y el manejo y control de inventarios, además de la disponibilidad de recursos (capacidad instalada). Las limitantes que principalmente se presentan al implementar una herramienta logística son financieras, físicas y tecnológicas. Aunque existen unas limitantes que ocasionan que la implementación de herramientas logísticas sea cada vez menor, se debe tener en cuenta que el contexto estimula a los directivos a emprender proyectos de mejora en hospitales (Amaya et al., 2010). Eso indica que entre más conozcan los miembros de las entidades de salud su entorno y lo que necesitan comparándola con otras instituciones, mayores serán las oportunidades para cambiar y alcanzar así niveles elevados de eficiencia en la atención hospitalaria.

Con respecto a la organización de bodegas y al manejo y control de inventarios, la literatura demuestra que en la gestión de inventarios es esencial la colaboración obtenida entre el proveedor y el comprador, que generalmente se encuentra sometida a políticas que cada entidad debe definir dependiendo de la conveniencia y acuerdos entre las partes. Aquí lo importante es operacionalizar la relación de tiempo-espaciopatrones que permitan identificar aquellos logros o fallos del sistema. Este es uno de los pilares para el óptimo desarrollo de la logística hospitalaria. En la encuesta se evidencia la necesidad de invertir mayores esfuerzos en este aspecto.

En definitiva, Barranquilla es una ciudad que desde su contextualización es posible notar que existe mucho por hacer en el tema de salud específicamente en el tema de la logística hospitalaria. Los hospitales, IPS y clínicas como primer paso deben reconocer la necesidad de implementar herramientas que permitan evolucionar el sistema logístico para ser cada vez más competentes.

\section{RECOMENDACIONES}

Se propone iniciar con una cultura de apertura que permita a los integrantes y directivos implementar herramientas logísticas para propiciar mejoras sustanciales especialmente en el área de organización de bodegas y control de inventarios. Además, es de alta relevancia establecer políticas adecuadas con los proveedores. Este último factor corresponde al análisis de la cadena de suministro que está relacionado con la gestión de inventarios, que varía según el funcionamiento logístico (Jiménez et all, 2007). Es entonces, que, si se realiza una mejora en la cadena de suministros, también se facilita con ello el manejo y control de inventarios o viceversa. Esta cadena de suministros abarca desde el fabricante, distribuidor, almacén o farmacia, unidades hospitalarias y por último el paciente. Esto quiere decir, que para el manejo de inventarios se debe tener en cuenta los tiempos para tener el medicamento disponible en farmacia para la entrega a tiempo del paciente y con base a ello establecer la política más beneficiosa. Importante también mencionar que conforme y las farmacias seas propias tal y como lo demostró la encuesta en el $48 \%$ de las entidades de salud entrevistadas, mayor será el control sobre los insumos y medicamentos. Sin embargo, las farmacias subcontratadas ofrecen una ventaja porque le permiten a las Entidades de Salud dedicarse a otras áreas sobre las cuales tienen mayor conocimiento.

La entidad no solo debe implementar el manejo y control de inventarios sino también el análisis de la cadena de suministros, donde obtendrán mayor éxito en las mejoras de logística hospitalaria de sus Centros de salud.

Se le agradece la participación en el proyecto de investigación del que se deriva este artículo a la estudiante Danna Betancour.

\section{REFERENCIAS.}

[1]. Ozores, B. (2007). Logística hospitalaria claves y tendencias de las operaciones logísticas en el sector hospitalario: calidad en la atención sanitaria y reducción de costes. Editorial Marge Book. España. Vol 1. Pp 129

[2]. Beaulieu, M.; Landry, S. (2002). "Comment gérer la logistique hospitalière? Deux pays, deux réalité", Gestion, vol. 27, $\quad \mathrm{n}^{\circ} 3, \quad$ p. 9198.DOI:10.3917/riges.273.0091

[3]. Hernández, P., Velasco, N \& Amaya C. (2008). Modelo de coordinación de inventarios en la cadena de abastecimiento de medicamentos de un hospital público. Los cuadernos de PYLO. Recuperado de http://dspace.uniandes.edu.co:9090/xmlui/bitstream/h andle/1992/1080/PYLO\%20H\%202008\%204.pdf?se quence $=4$

[4]. Ballou, R. (2004). Logística: administración de la cadena de suministro. Editorial Prentice Hall. Mexico. Pp 370.

[5]. Bustamante, M \& López, L. (2005). Abastecimiento eficiente: el efecto cola en los hospitales1. Revista 
Estudios Seriados en Gestión de Instituciones de Salud Centro de Gestión de Instituciones de Salud, Universidad de Talca. Volumen 1. Recuperado de http://mggp.utalca.cl/docs/taller_de_salud_publica/Ef ecto_Cola_en_los_hospitales.pdf

[6]. Gonzabay Cabrera, j. $\quad$ D. R., \& Parra Palacios, G. B. (2013). Calidad de Atención de Enfermería del Área de Hospitalización del Hospital "Dr. Rafael Serrano López” La Libertad 2011-2012 (Disertación Doctoral).

[7]. Ivan Su, S. I., Gammelgaard, B., \& Yang, S. L. (2011). Logistics innovation process revisited: insights from a hospital case study. International Journal of Physical Distribution \& Logistics Management, 41(6), 577600.

[8]. Organización Mundial de la Salud, (2014). Estadisticas Sanitarias Mundiales. Ediciones OMS. Suiza. Pp 130-138

[9]. Secretaria Distrital de Salud, (2013). Informe de Gestion Enero-Abril 2013. Pp 92-94 